\title{
Germanica
}

\section{Ilse Aichinger. Le recours discret au conte dans une écriture de l'étrangeté}

Der unauffällige Einsatz des Märchens in Ilse Aichingers Prosa der Entfremdung. The discreet recourse to narrative in strangeness writing.

\section{Brigitte Desbrière-Nicolas}

\section{OpenEdition}

\section{Journals}

Édition électronique

URL : http://journals.openedition.org/germanica/1049

DOI : 10.4000/germanica. 1049

ISSN : 2107-0784

\section{Éditeur}

Université de Lille

\section{Édition imprimée}

Date de publication : 30 juin 2010

Pagination : 41-60

ISBN : 9782913857254

ISSN : 0984-2632

\section{Référence électronique}

Brigitte Desbrière-Nicolas, « Ilse Aichinger. Le recours discret au conte dans une écriture de

l'étrangeté ", Germanica [En ligne], 46 | 2010, mis en ligne le 01 juin 2012, consulté le 06 octobre 2020

URL : http://journals.openedition.org/germanica/1049; DOI : https://doi.org/10.4000/germanica.1049

Ce document a été généré automatiquement le 6 octobre 2020.

(c) Tous droits réservés 


\title{
Ilse Aichinger. Le recours discret au conte dans une écriture de l'étrangeté
}

Der unauffällige Einsatz des Märchens in Ilse Aichingers Prosa der Entfremdung. The discreet recourse to narrative in strangeness writing.

\author{
Brigitte Desbrière-Nicolas
}

1 À l'automne 2004, Ilse Aichinger publie aux éditions Korrespondenzen un petit livre au titre insolite, car ancré dans toutes les mémoires enfantines grâce aux frères Grimm: Der Wolf und die sieben jungen Geißlein (Le loup et les sept chevreaux). Curieusement, il se glisse au milieu d'une série de publications de facture nouvelle qui recueillent les chroniques hebdomadaires rédigées, après de longues années de silence, pour deux quotidiens viennois, Der Standard (d'octobre 2000 à décembre 2004) et Die Presse (à partir de janvier 2005). « Der Wolf und die sieben jungen Geißlein » de 2004 est la reprise de la contribution d'Ilse Aichinger à un ouvrage collectif édité en 1974 par Jochen Jung², Märchen, Sagen und Abenteuergeschichten auf alten Bilderbogen neu erzählt von Autoren unserer Zeit (Contes, légendes et récits d'aventures de recueils anciens illustrés, réécrits par des auteurs de notre temps) ${ }^{3}$. La précision de l'éditeur soulignant la liberté totale laissée aux contributeurs quant au choix du texte et au mode d'appropriation, manifeste à l'évidence l'intérêt présenté par une réédition qui lui est exclusivement consacrée. La minceur de l'ouvrage (vingt-cinq pages), sa composition sans surprise, ne permettent pourtant pas de conclure à sa limpidité. Si le fait d'attribuer aux frères Grimm la qualité d'auteurs et de confier à $\mathrm{S}$. Fässler la rédaction d'une postface n'étonne guère, les deux textes d'Ilse Aichinger suscitent en revanche perplexité et curiosité en raison de la superposition de différentes strates temporelles révélatrices des pesanteurs qui ont habité l'écrivain tout au long de son existence. Le propos introductif daté de septembre 2004, «Das grüne Märchenbuch aus Linz» («Le livre de contes à la couverture verte de Linz»), évoque la petite enfance. Tant par le dit que par les silences, il constitue avec la postface un cadre qui ouvre deux perspectives de lecture complémentaires. Quant à la reproduction du conte populaire de la tradition, elle ne 
confine pas le texte des Grimm dans une fonction conventionnelle: les légers gauchissements introduits subrepticement en font l'annonciateur discret de l'orientation de la réécriture qui est au cœur de la publication.

2 À partir des investigations menées sur la mise en œuvre du conte non seulement dans cet ouvrage tardif, mais aussi dans le roman de jeunesse consacré aux années de ténèbres vécues à Vienne par l'adolescente demi-juive, où apparaît un Chaperon rouge aux contours étranges ${ }^{4}$, le présent article propose une approche renouvelée de l'écriture d'Ilse Aichinger, une écriture qui reflète le regard de l'étrangeté que l'écrivain jette sur le monde et qui saisit les émergences fragmentées des images obsédantes gravées dans la mémoire.

\section{Du premier livre de contes à la réécriture distanciée d'un conte}

C'est sur un conte d'animaux connu depuis l'Antiquité que se fixe le choix d'I. Aichinger en 1974. Les animaux doués de la parole y sont des personnages à part entière dont les comportements sociaux et les structures familiales sont calqués sur les sociétés humaines. Alors que certains chercheurs rangent cette catégorie de contes parmi les fables, Max Lüthi ${ }^{5}$ établit une nette distinction entre les deux genres, la fable étant dès son origine conçue en fonction de sa dimension éducative, de sa morale clairement énoncée. Rien de tel dans la version d'I. Aichinger, ni même dans la reproduction du numéro huit du Bilderbogen für Schule und Haus (Recueil illustré pour l'école et la maison) de 1897 placé en regard (JJ, 8) ${ }^{6}$. Les enjeux sont ailleurs. Les remarques générales de Jochen Jung dans sa préface peuvent permettre de comprendre la prédilection de l'écrivain autrichien pour les frères Grimm. Même expurgés au fil des éditions, leurs contes restent en effet empreints de rébellion, de rêve, de merveilleux et d'utopie. Mais la clarification du choix de ce conte d'animaux passe quant à elle par une lecture attentive et ouverte du propos introductif.

\section{« Das grüne Märchenbuch aus Linz » (W7G, 5-8)}

4 La tonalité du propos au titre anodin est suggérée d'emblée par une juxtaposition hardie de couleurs: d'une part le vert du livre de contes fixé dans la mémoire, de l'autre l'orange vif des œuvres de Cioran qui brille sur la table grise du célèbre café viennois, le Café Demel. I. Aichinger y passe à l'époque quotidiennement de longues heures et jette sur le papier des observations sur tout ce qui s'offre à son regard, puis sert de point de départ au jaillissement des images enfouies dans la mémoire qui alimentent les chroniques hebdomadaires.

5 La mention du nom de Cioran suffit à signaler que le propos introductif n'appartient pas à la catégorie de l'exercice convenu de la préface banale, mais doit être rangé dans la série des chroniques qui lui sont contemporaines. En effet, les réflexions amères et désenchantées de Cioran émaillent chacune des contributions du Standard depuis le mois de mai 2004, à la suite de la mort accidentelle et brutale de Richard Reichensperger, l'ami encore jeune, journaliste et spécialiste de littérature qui, en lui ouvrant les colonnes du journal Der Standard, a fait sortir I. Aichinger d'une longue période de silence ${ }^{7}$. 
6 Trois aphorismes de Cioran, «l'un des témoins à charge les plus hardis et les plus violents, jamais apparu au cours du procès interminable engagé entre les hommes et le monde ou Dieu $»^{8}$ structurent « Das grüne Märchenbuch aus Linz ». Le premier donne le ton, «les inconsolations de toute sorte passent, mais le fond dont elles procèdent subsiste toujours » $(\mathrm{W} 7 \mathrm{G}, 5)^{9}$. Il introduit les champs lexicaux dominants dans un texte qui, à l'arrière-plan, fait entrer en résonance l'épreuve présente d'une séparation douloureuse et les souvenirs d'une époque lointaine où surgirent les premières interrogations sur les souffrances générées par le simple fait d'être au monde. Etablir un lien entre le tourment d'exister et le premier livre de contes ne doit rien au hasard. Comme l'observe Marthe Robert: « Par le réquisitoire qu'il laisse percer sous la façade de sa moralité officielle, le conte se rattache assurément au vieux fond commun des mythes et légendes où l'humanité archaïque a déposé son horreur de naitre ${ }^{10}$.

7 Le livre vert qui accompagne la petite enfance, est présenté comme un dispensateur de consolations provisoires dont l'importance s'est accrue au fil des jours sans jamais être démentie. Pourtant la description brève qui en est faite se limite à un relevé objectif de quelques détails à connotations restrictives: le nombre des contes est restreint, ils ne sont pas présentés dans leur version originale et la dorure du titre s'écaille... En dépit de son ambivalence, la couleur verte - tantôt couleur de l'espérance, de la nature et de l'apaisement, tantôt couleur du diable et de l'étrange ${ }^{11}$ - doit quant à elle être reçue plutôt positivement car elle est associée un peu plus tard à «la forêt verte du livre vert » (W7G, 6) incluse dans la liste des agents potentiellement protecteurs. La place du livre sur le plancher de la chambre le destine à être essentiellement un objet d'appel puisque, conformément à la tradition, "les contes [sont] racontés». Mais si en apparence le cérémonial du conte est respecté, l'utilisation de la forme passive sans désignation d'agent, puis l'emploi du sujet indéfini («quel que soit» le conteur) font apparaître en creux une situation de manque relationnel. L'heure du conte n'est pas «un moment de respiration commune ${ }^{12}$, elle n'a rien de cet "événement interpersonnel où l'adulte et l'enfant prennent une part égale $»^{13}$ dont B. Bettelheim, citant le cas de Goethe et de sa mère, souligne l'influence décisive sur le développement du jeune auditeur. Une autre observation formulée par le psychologue, selon laquelle « l'enfant expérimente l'ordre du monde à l'image de ses parents et de ce qui se passe à l'intérieur de la famille» (PCF, 69), pourrait par ailleurs expliquer la peinture radicalement négative de Linz, la ville de la petite enfance.

8 La description du Linz "de cette époque là ", cristallisation de tous les déficits, est marquée du sceau de la subjectivité absolue. Elle est constituée de quelques traits caractéristiques et exhale l'enfermement. Ce ressenti est imputable au paysage de fleuve et de montagnes dont la trop grande proximité entrave la dimension consolatrice potentielle. L'enfermement est manifesté par des notations visuelles et auditives rapides limitées à deux rues dont l'activité humaine est absente, à l'école aux couleurs criardes, à une église dont les cloches ne font entendre qu'un tintement et à la cathédrale qui, comme beaucoup de choses à Linz, est plutôt ratée. Une impression de confinement intolérable s'impose d'autant plus au lecteur que le mot «Linz» est martelé jusqu'à l'excès dans les quelques lignes qui encadrent les énumérations évoquant l'espoir déçu de trouver dans les personnages et le décor des contes une chance de consolation durable. L'ombre projetée par la ville sur les récits de fiction emprisonne les héros dans leur quête et étouffe leur vocation de passeur d'espoir face aux épreuves de l'existence. Rien d'étonnant à ce que soient successivement cités des 
êtres jeunes, les chevreaux et le Chaperon rouge, Blanche-Neige, Hänsel et Gretl et Raiponce dans sa tour. Les uns sont menacés par le prédateur, les autres sont soit exposés à la méchanceté obstinée d'une marâtre, soit torturés par l'angoisse de l'abandon, ou encore condamnés à la captivité, victimes du comportement impulsif d'un couple de parents inconséquents.

« Das grüne Märchenbuch aus Linz » prend ainsi la forme d'un réquisitoire distancié contre les expériences vécues au cours de la petite enfance. La voix qui porte l'accusation présente la curieuse particularité d'être plurielle, c'est celle d'un « nous ». Elle est en outre relayée par celle indéfinie $d u$ "on », indice d'un déficit identitaire. Intercalé entre deux passages laconiques où défilent les héros du livre, ce «nous » non explicité ne prend vie que par l'évocation d'un détail anecdotique, un geste qui se fige bientôt dans une interrogation béante sur l'ennui, le vide irrémédiable de l'existence. Il s'agit de l'expérience partagée par les sœurs jumelles Ilse et Helga qui tentent vainement de tirer vers le bas des cheveux trop courts. Geste de repli autoprotecteur? Simulacre de disparition?

En dépit de l'omniprésence de Cioran, l'un des rares penseurs qui selon I. Aichinger soit capable de montrer sous son vrai jour, sans enjolivures ni lamentations le tourment d'exister, c'est vers les frères Grimm que l'écrivain se tourne fort logiquement à la fin de son propos. Faisant preuve d'un "anarchisme moins radical», ils ont su collecter tout ce qu'offre le monde, tant dans le règne minéral, animal et végétal que dans la sphère humaine, avec son lot de niaiseries et de pièges et son cortège de personnages qui y sont confrontés. Toutefois, c'est sans candeur naïve qu'ils lèguent au peuple le fruit de leur labeur. Mais Cioran ou les Grimm ne sont plus là pour prodiguer leurs conseils... Alors en guise de conclusion, I. Aichinger dévoie l'ordre établi du conte du loup et des chevreaux et confie à l'élément minéral, accessoire de la délivrance des victimes, le soin de transmettre le message qui fonde le propos introductif: «Il ne reste plus que les sept pierres au fond de la fontaine, elles seules sont capables de faire face au tourment d'être au monde » (W7G, 8).

\section{Le conte des frères Grimm}

Faire précéder la réécriture du conte de la transcription du texte source n'est pas en soi exceptionnel. Pourtant il convient de s'attarder sur la version dite de Jacob et Wilhelm Grimm dans l'opuscule, et de la comparer à celle qui a été établie par leurs soins après l'ultime révision pour l'édition de $1857^{14}$. De prime abord la reprise est fidèle, pourtant la curiosité du lecteur est éveillée par deux austriacismes ${ }^{15}$ et surtout par le recours à la graphie moderne de « rau(h) " pour qualifier la voix du loup. Le choix est d'autant plus surprenant que l'auteur rejette la réforme de l'orthographe pour ses propres écrits. La présentation typographique attire également l'attention. Aux six paragraphes compacts du texte du dix-neuvième siècle dont le schéma ne se distingue en rien de la construction d'un conte merveilleux ${ }^{16}$ (1. situation initiale: éloignement de la mère et interdiction, 2. persuasion trompeuse de l'agresseur, 3. transgression de l'interdiction, 4. méfait, 5. constat du méfait et quête de la mère, 6. délivrance des victimes, 7. punition du loup), I. Aichinger substitue vingt et une unités narratives séparées par une ligne qui correspondent aux microphrases de l'action. Un nombre non négligeable de modifications infimes provoque une accélération du rythme et suscite une concentration de l'intérêt sur les scènes de face à face entre les principaux acteurs. Le 
style adopté par les Grimm qui manifeste une prise de distance par rapport à l'oralité pure, est remodelé par la juxtaposition de phrases plus courtes et par une ponctuation porteuse d'une expressivité accrue, mieux adaptée à la teneur des échanges. S'y ajoutent le remplacement fréquent des pronoms personnels par la répétition clarificatrice des noms des protagonistes et l'élagage des affleurements d'un enrichissement formel. Cette liste non exhaustive souligne l'intensité accrue du regard posé sur l'action. Contrastant avec le manque relationnel stigmatisé dans le propos introductif, l'accentuation de la dimension affective vécue dans le cadre de la relation maternelle est frappante. Elle s'opère tantôt sur le mode de l'expansion, tantôt sur celui de la réduction. On y trouve les paroles attentives d'une mère inquiète, mais la délivrance des chevreaux n'est accompagnée que de l'écho du soupir de soulagement: «ils étaient sains et saufs».

Quant aux transformations opérées dans la séquence évoquant le rôle décisif joué par le troisième auxiliaire du prédateur lors de la préparation du méfait, elles se chargent d'une signification toute particulière. Elles concernent d'abord la transcription des pensées du meunier qui, avant de céder finalement à la menace, refuse d'abord sa collaboration. Le discours direct qui ne laisse pas de zone d'ombre est employé dans le conte traditionnel: « Le loup va tromper quelqu'un ». Il cède la place à une formulation plus diffuse au discours indirect qui contraste singulièrement avec la forme dominante adoptée, celle de la concentration et de la concision. En outre la disparition de l'exclamation lucide teintée de résignation qui clôt le récit du face à face dans la version de 1857: "Ainsi sont les hommes!", laisse entrevoir une pratique de détournement du texte existant qui vise à la dénonciation par l'atténuation apparente et le commentaire retenu. Les glissements observés au niveau de la dénomination du loup s'inscrivent dans la même perspective. Si le responsable du massacre est, dans l'édition de référence, désigné à deux reprises par les termes de " monstre » et d'animal « impie ", c'est-à-dire classé dans les créatures diaboliques marginalisées, I. Aichinger lui attribue le titre de "méchant " l'intégrant ainsi dans la sphère du mal qui habite le monde créé. Ainsi I. Aichinger ouvre-t-elle insidieusement la voie à un mode de perception du monde existant qui se déploie dans la réécriture du conte portant sa signature.

\section{La réécriture du conte}

La présentation typographique de la version d'I. Aichinger dans l'opuscule, une série de paragraphes courts séparés par une ligne blanche ${ }^{17}$, reprend celle adoptée pour le conte des frères Grimm. Elle est parfaitement adaptée à la forme d'un texte éclaté, caractérisé par l'absence d'une instance narrative et qui, à la façon d'une pièce de théâtre contemporaine, laisse successivement la parole aux protagonistes de l'histoire. Prédateur, complices, victimes proposent au lecteur une approche rétrospective de l'événement funeste, laissant dans l'ombre le dénouement et le châtiment.

14 La réécriture du « Loup et [des] sept chevreaux » prend ses racines dans l'expérience de la proximité immédiate de la mort, dans cet instant extrême où s'éclaire pour la créature la relation qu'elle entretient avec le monde. Ce travail sur le conte s'inscrit dans une pratique poétique dont les fondements ont été exposés au travers de textes de fiction ou de réflexion composés dans l'immédiat après-guerre. "Le récit à notre époque » énonce avec clarté la démarche qui s'est imposée à la survivante du génocide: 
La forme n'est jamais née d'une sensation de sécurité, mais au contraire toujours quand la fin était en vue. [...] Si nous nous y prenons bien, nous pouvons retourner ce qui semble tourné contre nous; nous pouvons précisément entreprendre de raconter depuis la fin et jusqu'à la fin, et c'est de nouveau pour nous l'aube du monde ${ }^{18}$.

«Le loup et les sept chevreaux » se compose de la juxtaposition soigneusement agencée de treize témoignages. Il s'ouvre sur la « déposition » du loup introduite par la formule reprise par tous les intervenants : "Je suis... ». Elle concerne le moment qui précède le méfait, alors que le "méchant», comme le nomment les enfants du village avec effroi, chemine sur les anciennes coulées. Il ne sait pas que les villages et les chevreaux traversent les anciennes coulées... Cet argument de disculpation n'insinue-t-il pas que les distinctions entre la sphère humaine et le règne animal sont superficielles? Puis se succèdent les voix de trois chevreaux dont l'identification repose sur l'ordre de leur venue au monde. Leur présentation est lapidaire. Ils ont ceci en commun que les données de la naissance, fussent-elles positives ou négatives, déterminent le choix d'une cachette bien illusoire: le chétif se cache dans le poêle qui lui dispensait jusque-là une chaleur protectrice, le plus intelligent, abusé par ses connaissances, pénètre dans l'armoire et le préféré de la mère, sans doute habitué à la facilité confiante, pense que la nappe de la table suffira à le cacher. Pas de commentaires, pas de lamentations à propos de l'épreuve subie, simplement une question rétrospective chez le faible sur le comportement hésitant du loup à son égard, ou chez le cadet une prise de conscience des limites de l'intelligence, ou bien encore une interrogation moins anodine chez le bien-aimé sur le pouvoir réel de l'amour.

Désormais les voix des victimes alternent avec celles des autres protagonistes. Le tournant s'amorce avec la déclaration de l'homme complice, le meunier. La reprise littérale de la formulation de 1857 n'est pas fortuite: «Le loup va tromper quelqu'un ». La stratégie de défense alors mise en œuvre n'a pas besoin de l'indignation d'un commentateur extérieur pour rendre intolérable l'artifice destiné à masquer la lâcheté humaine funeste: la compassion à l'égard du loup est invoquée, puis érigée avec assurance en principe ayant valeur générale. En revanche pas un mot du boulanger, le seul peut-être à s'être montré véritablement compatissant.

Un peu plus tard, l'autre complice, l'épicier, banalise par quelques affirmations brèves son implication dans le méfait. D'ailleurs la voix du chevreau, le deuxième de la famille, qui s'intercale entre celle des deux hommes, semble relativiser la responsabilité des auxiliaires du loup puisqu'il affirme la conscience qu'il a de son insignifiance. Il rit de l'arrivée du loup, ne se cache pas, saute simplement dans son lit. Il connaît la vanité de l'existence individuelle et sait que par cette épreuve les autres l'apprendront aussi. Le leitmotiv de l'insignifiance, aux accents de comptine, figure d'ailleurs, écrit de la main d'I. Aichinger, au bas de la page de couverture de l'opuscule: « Après un, vient deux et après deux, vient trois, on le sait » (W7G, 20).

L'intervention du sixième chevreau à la suite de l'épicier, puis ultérieurement celle du quatrième, confirment que le lecteur se trouve ouvertement en présence des porteparole de l'écrivain. La découverte de l'égoïsme des autres membres de la fratrie, chacun étant soucieux de sa propre survie, fait jaillir la colère chez le sixième, une colère positive puisque jusque-là ardemment désirée: « être suffisamment en colère, je l'ai souhaité depuis toujours " (W7G, 20). Dans cette phrase résonne un écho lointain des passages nombreux dans l'œuvre d'I. Aichinger qui célèbrent la colère comme arme de lutte efficace contre l'optimisme béat des hommes de ce temps et contre les 
préjudices causés par l'acceptation spontanée du monde tel qu'il est. Le quatrième chevreau partage quant à lui avec les jumelles du propos introductif le sentiment d'ennui, ce qui l'amène à imaginer l'histoire du loup et à se rendre simplement dans la cuisine pour la terminer avant de se faire dévorer. C'est aussi dans la cuisine d'une amie qui l'hébergeait temporairement avec sa mère que la jeune Ilse entreprit en 1945 la rédaction de son roman Die größere Hoffnung (Le plus grand espoir), cette fresque étrange où les échappées dans l'imaginaire permettent à Ellen, l'enfant demi-juive solitaire, de vivre debout malgré la menace et les persécutions.

La mère quant à elle se défend d'être une vieille femme comme dans le conte populaire, elle n'est pas davantage la "sage-femme " des contes merveilleux de l'espace germanique, elle refuse qu'on puisse voir en elle autre chose qu'un être ballotté entre la détresse et le bonheur. Le chevreau le plus jeune, le survivant, au demeurant peu prolixe, ne perçoit de la mère que la détresse: « elle était noire à force de chercher. Comme le loup » (W7G, 21).

20 Seule la neuvième prise de parole laconique et insolite évoque allusivement la noyade du loup: la voix de la fontaine impassible se fait entendre, simple élément du décor, elle a malgré elle contribué à renverser le cours des choses.

21 Le mot de la fin revient à la pierre. L'observation qui conclut le texte d'entrée, l'a en quelque sorte introduit: «Je suis la première pierre. Je ne suis pas venue au monde. Les six autres non plus » (W7G, 21).

Les remarques pertinentes de S. Fässler dans la postface (W7G, 23-25) orientent l'interprétation vers l'établissement d'un parallèle entre les circonstances du massacre dans le conte d'animaux et les épreuves vécues à Vienne par la jeune fille demi-juive qui a connu les pièges que recèlent les cachettes, les limites de la solidarité dans les situations extrêmes, et la difficulté de distinguer le loup des brebis... Mais il s'avère impossible de ne pas mettre au premier plan les enjeux existentiels de la réécriture de l'histoire du massacre. Ce n'est pas un hasard si dans une chronique de 2005 consacrée aux paysages de l'existence ${ }^{19}$, il est question du loup et des chevreaux pour évoquer non seulement les transformations du regard des chevreaux à l'issue de leur plongée dans les ténèbres étouffantes mais aussi les interrogations relatives au silence d'un Dieu lointain. Les perspectives ouvertes par les réflexions des deuxième, sixième et quatrième chevreaux doivent être lues comme le prolongement d'une notice du journal de $1973^{20}$, pratiquement concomitante de la rédaction du conte pour l'album de J. Jung: «Je ne peux plus m'accommoder du fait d'être née » (KMF, 81). Un tel état d'esprit laisse son empreinte dans les propos prêtés à la mère livrée au désarroi, car au fond, la fin heureuse du conte de la tradition ne signifie-t-elle pas plutôt une seconde mise au monde, une nouvelle façon de livrer ses petits aux tourments de l'existence? I. Aichinger écrit en 1964 dans son Journal: «Définition des mères: la possibilité de n'être pas au monde » (KMF, 73). Cette note invite à se tourner à nouveau vers « Das grüne Märchenbuch aus Linz » pour explorer au travers de textes autobiographiques les silences enchâssant les allusions à la constellation familiale pendant la petite enfance. 


\section{Les données initiales de l'existence et l'univers du conte. Convergences} ombres de Linz, et où ne se dessine aucun visage. Et pourtant deux silhouettes de l'époque, frappées du sceau de l'étrangeté, se détachent sur la toile de fond des souvenirs fragmentés qui hantent les chroniques tardives. Celle du père d'abord, un enseignant fantasque. Il est indissociable du livre des contes de Grimm, le premier, le préféré trouvé parmi les cadeaux de Noël sous le sapin qui comme d'habitude n'avait pas été payé (UR, $78 ; 120)$ par celui qui s'enfonce dans l'endettement pour acquérir des exemplaires identiques d'œuvres complètes et qui finit temporairement par être interné (UR, 21). Il n'hésite guère en 1927 à privilégier sa passion pour les livres et à accepter la séparation du couple ${ }^{21}$. La relation entretenue avec l'homme qui exerça à « court terme » sa responsabilité paternelle semble trouver un écho dans une remarque relevée dans l'article du 20.12.2002 intitulé « Abschied von Weihnachten" («L'adieu à Noël », UR, 78-79). Elle concerne la prédilection des jumelles pour un conte merveilleux, tiré du premier livre de Grimm reçu en cadeau: «Die Gänsehirtin am Brunnen» («La gardeuse d'oies à la fontaine», MG, 521-528)22, un titre qui curieusement n'est pas mentionné dans la préface de 2004. L'organisation du récit bouscule celle observée par V. Propp et relègue la relation du méfait au milieu du récit, alors que l'action contraire, initiée par l'épreuve infligée par une " sorcière » à un jeune comte est déjà fort avancée. Au centre de l'intrigue, la colère d'un père, un roi blessé dans sa vanité par l'incapacité de sa plus jeune fille à traduire en paroles l'amour qu'elle lui voue. Il la chasse impitoyablement et l'expose aux périls de la forêt profonde. L'épilogue heureux est l'œuvre d'une "sage-femme» dissimulée sous les traits de la sorcière qui pourvoit simultanément à la protection de l'adolescente, en la transformant temporairement en gardeuse d'oies, et à la punition du père-bourreau condamné trois ans durant à l'angoisse. Sur le malheur né de la cruauté du père, la chronique de 2002 garde un silence éloquent. Elle s'attarde en revanche sur l'engouement pour le conte, résumé en une phrase gravée dans la mémoire des jumelles: «Alte, du wirst unverschämt » (« Vieille femme, tu deviens insolente »). Elle ne les quittera plus. De l'aveu même de l'écrivain, elle s'avère être une clé permettant de « rester sur les traces de l'enfance» (UR, 79), de préserver ce regard qui ne se fige pas dans un mode de perception préétabli ${ }^{23}$. Mais la réplique n'est-elle pas devenue aussi pour la chroniqueuse polémiste une façon de se définir en référence à la « sagefemme » du conte? Quoi qu'il en soit, c'est au niveau du non-dit que, dans l'évocation de la "gardeuse d'oies à la fontaine ", l'ombre des conflits irrésolus liés à la personne du père se manifeste ${ }^{24}$.

Alors que la mère qui est médecin ne laisse aucune trace dans les textes relatifs à cette période, la gouvernante des enfants, la « demoiselle schizophrène » de Linz s'affiche en revanche au premier plan. Le but favori de ses promenades avec les fillettes est l'hôpital psychiatrique provincial dont elle est sortie prématurément (UR, 102). Dans la prairie qui jouxte l'établissement, les jumelles goûtent l'opportunité d'échapper aux querelles familiales. Mais elles vivent alors aussi l'expérience initiale d'un «voyage invraisemblable » (UR, 21), les poignets marqués par la main de fer d'Emma Schrack dont l'étrangeté suscite des interrogations retenues qui s'enracinent profondément 
dans la mémoire (UR, 102; 121). Elles s'ajoutent à celles générées par la naissance gémellaire.

Dans un entretien, I. Aichinger déclare en 2003 à ce propos:

Je considère que mon existence est dépourvue de toute nécessité [...] - enfant j'ai déjà ressenti le fait d'exister soudain comme une absurdité imposée. [...] Dès le commencement ce fut difficile, parce que j'ai une sœur jumelle. Et alors on ne vous perçoit pas comme un individu. D'ailleurs nous nous ressemblions tellement que même notre mère ne pouvait pas nous distinguer l'une de l'autre. Pourtant nous sommes fondamentalement différentes comme des personnes étrangères (UR, $181)^{25}$.

Le malaise originel ne peut être évoqué plus nettement. Les dommages causés par la perception des autres, empreinte de curiosité et d'indifférence ${ }^{26}$, façonnent le regard que les jumelles portent sur elles-mêmes et sur l'existence. Le commentaire accompagnant une photo de vacances de l'été 1928, «Zwei in einem Boot » («À deux dans un bateau », FV, 110-111) dépeint avec une froideur distante (utilisation de la troisième personne du pluriel) l'état d'esprit des fillettes, caractérisé par la résignation désabusée et un sentiment de non-appartenance qui fait naître le rêve de «n'accoster nulle part ».

Pourtant le divorce des parents en 1928 offre l'opportunité d'un point d'ancrage. C'est Vienne et la découverte du lieu habitable par excellence, l'appartement de la grandmère maternelle dans la Hohlweggasse 1. Le titre énigmatique du recueil Kleist, Moos, Fasane (Kleist, Mousse et Faisans) qui fait référence à des noms de rues, circonscrit l'espace où ont été vécues les années heureuses de l'enfance. Le récit autobiographique éponyme de 1959, le premier du livre, exalte la cuisine, un lieu d'exception situé à la croisée de l'orient et de l'occident, un lieu d'échanges et de projets qui se met à vibrer au passage des trains et offre une vue sans pareille sur un paysage "riche de promesses » où domine le vert:

Je me souviens de la cuisine de ma grand-mère. [...] Tout comme l'on se souvient encore, même pendant le jour, de la lumière qui baignait les rêves, je me souviens de sa lumière aujourd'hui, quand elle m'apparaît comme un rayon de soleil sur une mer inconnue (KMF, 13).

Vienne, c'est aussi l'école du Sacré-Cœur où pour un temps les jumelles oublient les pesanteurs nées des questions sans réponse sur l'existence, où elles se libèrent des assauts soudains de l'ennui et de la peur $(\mathrm{S}, 64)$. Mais les années heureuses, parfois fugitivement assombries par le regard des voisins porté sur les «juives» (FV, 21), s'achèvent définitivement en 1939. Après le dernier Noël fêté dans la Hohlweggasse 1, où en 1938 toute la famille se trouve dans l'obligation de résider en raison des mesures raciales d'expulsion, sonne bientôt l'heure des séparations définitives, celle de l'éclatement de la fratrie, puis celle de l'aryanisation de l'appartement de l'aïeule.

L'« histoire des séparations» (UR, 68-69) commence le 4 juillet 1939 avec le départ définitif d'Helga pour l'Angleterre ${ }^{27}$, le pays de ses rêves. Le jour de la réunion d'adieu dans l'appartement de la Hohlweggasse 1 (UR, 68-69) se pare encore des couleurs de l'espoir partagé de retrouvailles prochaines sur le sol anglais où se trouve déjà la sœur aînée de la mère, mais en réalité il s'inscrira bientôt dans la mémoire comme étant le jour où le sol s'est dérobé sous les pieds ${ }^{28}$. I. Aichinger devra attendre 1948 pour revoir sa sœur à Londres, mais les liens sont restés intacts comme en témoignent l'exergue du recueil de récits Eliza Eliza (1965), composé de deux vers en langue anglaise tirés d'un poème d'Helga Michie(-Aichinger), puis deux textes consacrés à l'activité de graphiste 
de cette dernière, figurant dans l'édition de 1987 de Kleist, Moos, Fasane. Dans l'un d'entre eux, Die Linien meiner Schwester (Le graphisme de ma sœur), l'écrivain exprime sa fascination pour ces dessins où elle puise mystérieusement la consolation. La publication conjointe à Vienne en 2006, à l'occasion du quatre-vingt-cinquième anniversaire des deux sœurs, des livres Subtexte (Sous-textes) d'Ilse et Concord (Harmonie) ${ }^{29}$ d'Helga, manifeste s'il en était besoin un attachement indéfectible. Concord est la reprise du titre de l'une des neuf gravures à l'eau-forte de l'opuscule, où sont représentés des jumeaux dans une maison déchirée par une fracture...

Le premier septembre 1939, les assises de l'existence s'écroulent définitivement. Le statut de demi-juive assure certes provisoirement la survie et celle de la mère, mais ne protège pas de l'angoisse ni de la souffrance de voir voués à la déportation un à un tous les proches, dont la grand-mère chérie, le 6 mai 1942. Il s'agit peut-être alors pour l'adolescente moins de craindre la mort que de refuser la désagrégation de son être par la peur en fréquentant des groupes d'enfants persécutés, en trouvant refuge dans l'imaginaire comme l'invite à le penser la stratégie de la jeune héroïne du roman commencé par la survivante en 1945.

\section{Sur les traces du conte dans le roman}

31 Il n'est pas dénué d'intérêt d'étudier Die größere Hoffnung en explorant les traces laissées dans la fiction par l'univers du conte. D'ailleurs dans un texte de 1966, « Hilfsstelle » ("Centre d'aide », KMF, 28-31), l'auteur recourt à un conte pour évoquer brièvement sur le mode métaphorique Gretl, une adolescente lucide, consciente de son destin tragique inéluctable mais qui oriente son espoir vers d'autres horizons. Par le nouveau nom reçu au baptême, elle n'est plus l'hérö̈ne d'un conte qui sortirait un jour de la forêt obscure: «Ce n'était plus nécessaire de sortir des forêts pour retrouver le chemin de la maison, les forêts elles-mêmes y conduisaient. Les ténèbres avaient subi une métamorphose » (KMF, 30).

En dépit de son projet initial qui visait à faire la relation des faits tels qu'ils s'étaient déroulés, la jeune Ilse écrit Die größere Hoffnung, un livre dont les éléments réalistes ne sont pas transfigurés par l'imaginaire, mais dans lequel la peinture du vécu intérieur est baignée de sa lumière. Comme «tous ceux qui ont fait sous une forme ou sous une autre l'expérience de la proximité de la mort», Ilse Aichinger peut prendre «[son] expérience comme point de départ, afin de découvrir la vie à nouveau pour [elle]-même et pour d'autres $»^{30}(G, 10-11)$. Elle ne livre pas un document destiné à alimenter la chronique des faits historiques, mais un ouvrage qui garde la mémoire d'une résistance intérieure qui n'est pas l'habillage hypocrite d'un comportement de lâcheté mais une stratégie de survie de la dignité humaine face à l'idéologie de la mort.

\section{Le genre romanesque et le conte}

33 Le genre choisi, le roman, ne saurait surprendre si l'on considère comme M. Robert que le «faiseur de roman est un esprit épris de liberté, résolu à ne pas s'incliner devant l'irréversible » et caractérisé par son « déni subversif de la réalité immédiate » (RO, 36). Dans son exploration du monde du désir romanesque de changer la réalité, M. Robert en vient à s'intéresser à une imagination non écrite relevant essentiellement du psychique, celle qui conduit l'enfant à l'élaboration de son « roman familial », une fable 
biographique destinée à surmonter la crise qui survient lorsque l'idéalisation du couple parental se révèle être une chimère. Les observations formulées dans cette étude au niveau des convergences existant entre les intentions profondes du « roman familial » et celles du conte traditionnel de l'enfance, et au niveau de leur schéma général, se révèlent fructueuses si l'on considère Die größere Hoffnung comme une fiction située à la croisée de l'histoire d'une crise personnelle d'origine familiale et de l'Histoire, qui a fait sombrer le vingtième siècle dans la barbarie (MC, 16-17; RO, 82-100).

Des similitudes frappantes peuvent être constatées entre la structure du roman de jeunesse d'I. Aichinger et celle qui caractérise un conte merveilleux dont le héros est un enfant. Comme lui, la jeune Ellen connaît une enfance difficile, puisqu'elle est victime d'une faute $(M C, 17)$ antérieure à sa naissance (une malédiction due à l'ascendance maternelle juive). Comme lui, elle est exposée à une souffrance intolérable, provoquée par l'absence de la mère (contrainte à l'émigration). Quant au père, censé protéger des dangers du monde extérieur dans la famille traditionnelle, comme le souligne B. Bettelheim (PCF, 260), il la "maltraite » dans la mesure où il l'a abandonnée, lui a demandé de l'oublier (GH, 49). Il devient même un persécuteur (en tant qu'officier au service de l'idéologie d'exclusion et de mort qui sévit alors). Privée de la protection du père comme l'enfant du conte, Ellen ne peut compter que sur elle-même pour faire face au maléfice, et tenter sa chance dans le "vaste monde ", [celui] de la "forêt obscure où [elle] rencontre l'angoisse de la solitude » (MC, 17). Allant " de tourment en tourment ", elle doit elle aussi «forcer le passage à chaque étape de son développement » (RO, 88). La nécessité de pallier le manque fait naître chez Ellen l'ardent désir, voué à l'échec, de l'obtention d'un visa, ce qui déclenche une quête singulière initiée par le conseil d'un consul désemparé. Il engage la petite fille, par le biais d'un "objet magique ", un visa décoré de fleurs, d'oiseaux et d'étoiles que l'on s'accorde à soi-même, à être l'artisane de son propre sauvetage pour conquérir la liberté. Le roman n'emprunte pas que "l'objet magique " aux éléments-clés de l'analyse du conte merveilleux de Propp, il introduit aussi aux différentes phases de l'action des auxiliaires insolites issus du quotidien (un aveugle), de l'imaginaire religieux (François-Xavier, le roi David, Augustin, Noah) ou profane (Christophe Colomb), qui incitent à découvrir en soi et autour de soi les moyens de surmonter la peur, la solitude, et à croire quoi qu'il arrive en l'existence d'un ailleurs où continuera à briller le bleu de l'espoir.

Un épisode important dans le fonctionnement du conte (VP, 63-64), celui du héros transporté, conduit près du lieu où se trouve l'objet de sa quête, prend un relief particulier dans le roman (" Das heilige Land », « La terre promise »). « Le passage de la frontière" est un nouveau seuil qu'Ellen ainsi que le groupe d'enfants persécutés auquel elle s'est attachée, franchissent intérieurement, sur le mode onirique, au cours d'une équipée fantastique dans le corbillard d'un trafiquant de l'espoir. Si les acquis sont fragiles et vacillent sous les assauts réitérés du découragement et de l'angoisse générés par les menaces de plus en plus pressantes, ils permettent néanmoins d'affirmer la dignité des opprimés face aux "garçons en uniforme » ébranlés dans leurs certitudes ( $\mathrm{Im}$ Dienst einer fremden Macht», "Au service d'une puissance étrangère »), puis face aux agents des oppresseurs incrédules devant l'attitude résolue des enfants dénoncés («Das große Spiel », « Le grand jeu »). Après l'arrestation de la communauté des persécutés, c'est dans la solitude radicale qu'Ellen affronte au cours de la scène de "La mort de la grand-mère » l'épreuve du passage décisif qui s'opère par l'insertion directe de l'univers du conte. L'enfant tente alors de détourner du suicide 
son seul soutien familial, en l'implorant de garder la tête haute dans l'espoir à peine voilé de pouvoir elle-même résister au risque de la dissolution de son être. Avec l'énergie du désespoir, elle s'emploie à réveiller chez la vieille femme la conteuse des jours heureux de l'enfance, afin que l'aïeule puisse ainsi sauvegarder le fil ultime la rattachant à la vie. Mais la source est tarie et Ellen n'a plus d'autre issue que de se faire elle-même conteuse pour un public imaginaire constitué d'une grand-mère assoupie et de la nuit. Elle recrée l'histoire d'un Chaperon rouge qui est la projection du désir lancinant qui la consume de combler l'absence de la mère. L'héroïne du conte réinventé est coiffée d'un bonnet tricoté avec le fil rouge de la nostalgie de celle qui survit en exil, un bonnet qui a réussi à franchir l'océan et qui, telle une auréole incandescente et brûlante, la protège de la dévoration par le loup gris de la guerre. Mais l'imagination s'enlise bientôt dans la reprise aménagée du dialogue du conte avec le loup qui s'est substitué à l'aïeule. La conteuse s'engourdit dans un sommeil qui est habité par le rêve éphémère d'un déserteur désemparé, mais au moment du réveil se produit un passage qui correspond à un renversement du lien biologique: l'enfant désespérée et inflexible dans ses exigences se mue en un être plus mûr qui accepte, en tendant à l'aïeule le poison libérateur, d'être définitivement abandonnée. Mais ce passage introduit un dévoiement de la mise en œuvre du conte dans le roman. Certes, le livre s'achève effectivement à la façon d'un conte sur un "retour à la maison » accompagné d'une vision de l'adolescent tendrement aimé, Georg, l'interlocuteur privilégié dans le groupe des enfants persécutés et l'objet d'une quête nostalgique. Certes, le saut au-dessus du fleuve pour le rejoindre peut rappeler le franchissement de la rivière par l'entremise d'un canard blanc lorsque Hänsel et Gretl arrivent au terme de leur douloureuse aventure (MG, 72). Bien sûr. Mais c'est négliger les deux chapitres intermédiaires où s'engage la course échevelée de l'adolescente après le suicide de la grand-mère, une course qui est un élan préparant au saut délibéré qui est aussi celui de la mort au milieu des tirs d'obus. Au cours de cette phase d'errance, Ellen apparaît comme le prophète d'un ailleurs indéfini. Par sa présence insaisissable, ses silences éloquents, son étrange pouvoir de transformer en énigmes les questions et propos les plus banals, l'adolescente sème le trouble. Elle éveille obscurément l'esprit de rébellion dans une gare au moment du départ d'un train de munitions, puis dans un poste de police. Dans un cadre onirique affiché ("Flügeltraum », "Rêve d'ailes») l'héroïne réussit par un usage du langage paradoxal et de ses intervalles, à insuffler aux oppresseurs et à leurs sbires un grain d'humanité qui enraie temporairement les rouages d'un mécanisme bien rodé d'asservissement de la pensée. Puis la course folle se poursuit lorsque s'abat sur la ville un déluge de feu et de sang («Wundert euch nicht», "Ne vous étonnez pas »). La jeune adolescente se fraie un chemin au milieu d'une Apocalypse qui propage la mort dans les paysages familiers en provoquant l'effondrement d'un monde régi par des lois iniques. Mais contrairement à la perspective optimiste du conte, cette Apocalypse dessine aussi en filigrane les contours d'un monde à venir enfanté dans la douleur, où le « reste » ne se sera pas constitué de survivants qui seront la souche d'une humanité nouvelle, mais d'hommes et de femmes empressés de restaurer un ordre conforme aux pratiques des «loups » affamés de satisfactions immédiates et égoïstes (GH, 217). 


\section{De la mise en œuvre du conte dans le roman à sa réception}

36 "Le plus grand espoir", titre et terme d'un roman foisonnant, ne manque pas d'ambiguïté. Il forme avec la fin limpide et heureuse d'un conte traditionnel un contraste saisissant même s'il emprunte par ailleurs beaucoup au genre (sa structure générale, le mélange de réel et de merveilleux, sa capacité à proposer un horizon consolateur sans nier la misère elle-même), et même s'il fait ouvertement référence au conte $(\mathrm{GH}, 15,25,172)$ en introduisant le Chaperon rouge à un moment de crise extrême. Comme l'a montré Brigitta Oesch ${ }^{31}$, «Le plus grand espoir » est bien l'utopie d'une héroïne de conte qui ne se départit pas de la certitude d'une issue qui ressortit au merveilleux. Mais ici l'enjeu n'est pas tant de mener l'espoir à son terme que de le préserver dans la durée. Ce qui importe c'est de cheminer, guidé par un espoir que dessine l'emploi métaphorique de l'étoile. La confiance placée dans cette lumière de la nuit et la représentation imaginaire d'un monde meilleur nourrissent une force intérieure qui ne sauve pas la vie d'Ellen mais préserve sa dignité. Cette perspective singulière née d'une expérience vécue a suscité dans la critique un certain nombre de malentendus.

Malgré la place prépondérante prise dans l'imaginaire par les motifs et les références à l'Ancien et au Nouveau Testament ${ }^{32}$ dans le langage chiffré du récit, il ne saurait être question d'interpréter Die größere Hoffnung exclusivement comme un livre chrétien profondément religieux ${ }^{33}$, car ces emprunts sont eux aussi dévoyés à des fins subversives. Tout comme les éléments du conte, ils sont davantage mis au service immédiat de la créature désemparée confrontée à l'indicible horreur, que destinés à assurer le salut de son âme dans l'au-delà.

Quant à la question de la poétisation de la réalité vécue qui détournerait le regard de la barbarie, elle est posée par un courant de la réception contemporaine ${ }^{34}$. Négligeant les observations formulées par le jury de lycéens qui décerne en 1988 le Prix de Weilheim à I. Aichinger pour sa capacité à transmettre et à faire revivre l'histoire à des adolescents nés plusieurs décennies après la Seconde Guerre mondiale, des voix s'élèvent en effet pour mettre en accusation l'écriture de la survivante qui, « brouillant les cartes du réel et du rêve", s'avèrerait inapte à s'interroger sur la Shoah, ses causes et ses conséquences. Pourtant il est difficile de nier l'ancrage de l'œuvre dans la réalité. Ce serait faire peu de cas de l'omniprésence de la mention des multiples interdictions faites aux enfants juifs, de leur déportation, de l'obligation du port de l'étoile, de l'aryanisation des appartements, de la police secrète, de la nostalgie de l'autre rive. Par ailleurs, les critiques acerbes portant sur la compassion exprimée à l'égard des bourreaux qui ôterait aux Allemands le poids de la honte collective et relativiserait l'horreur, ne résistent guère à l'examen ${ }^{35}$. Ce que d'aucuns dénoncent comme la manifestation d'une grandeur d'âme intolérable corrobore pourtant l'analyse perspicace du phénomène de la soumission aveugle à l'idéologie nazie proposée par Albert Camus dans ses Lettres à un ami allemand ${ }^{36}$. À l'évidence le roman ne se livre pas à ces lecteurs qui le mesurent à l'aune d'attentes modelées par une grille de lecture préétablie et par une conception figée de l'engagement. Le déchiffrement patient de la scène de la mort de l'aïeule où surgit l'étrange Chaperon rouge suffit à réfuter l'affirmation de l'absence de réflexion profonde sur la Shoah. 
39 Ce que révèle ce passage-clé, ce n'est pas une désertion, une fuite dans l'imaginaire, c'est la caducité d'une tradition qui depuis Les mille et une nuits veut que la seule puissance du verbe empêche l'arrivée de la mort. Confronté à une réalité privée de sens, le pouvoir de la narration est ébranlé, et la violence aveugle élevée au rang de principe tue irrémédiablement le récit traditionnel et sa dimension salvatrice ${ }^{37}$. Voilà le message transmis au travers de ce conte moderne. Vouloir préserver de l'oubli la voix des victimes de la barbarie nazie passe nécessairement par la recherche d'une écriture nouvelle, à laquelle I. Aichinger consacrera sa vie.

\section{Ilse Aichinger et le conte, « ce genre qui joue sans cesse sur le caractère étranger et ambigu des choses familières » $(\mathrm{RO}, 102)$.}

La lecture ouverte des deux ouvrages étudiés, écrits à plusieurs décennies d'intervalle, fait apparaître que le conte populaire a laissé dans l'imaginaire de l'écrivain une empreinte profonde. Plus discret que les références à l'Histoire biblique, le conte apparaît néanmoins indéniablement comme l'un des éléments constitutifs de l'écriture d'une femme qui, en exploratrice de son temps, ne se propose pas de «faire découvrir le monde inconnu, mais de rendre étrange le monde par trop familier » $(\mathrm{KW}, 54)$ afin de transformer le regard de ses contemporains. Dès le roman de jeunesse, le conte est un instrument révélateur des ruptures consécutives à l'expérience des situations extrêmes provoquées par le cataclysme qui a ébranlé le vingtième siècle. Reçu dans l'enfance comme dispensateur de consolation, le conte permet par ailleurs d'exprimer les pesanteurs de l'intériorité et de dessiner par le biais de l'analogie le cheminement de l'individu confronté aux difficultés générées par la malédiction de l'existence imposée. Enfin, le recours à ce genre n'est pas un simple artifice poétique, une fuite devant la réalité comme pourraient le suggérer les affleurements du rêve et du merveilleux qui le caractérisent. Par l'éclairage singulier qu'il jette sur la réalité, il peut être mis au service du réalisme qu'I. Aichinger définit ainsi en 1984: «Un état ou une forme de vie, d'écriture ou d'action où l'exactitude intérieure et extérieure coïncident ». Et l'écrivain d'ajouter: «Ce que l'on appelle littérature engagée est aussi nécessaire. Mais l'engagement seul, sans la langue ne suffit pas $\aleph^{38}$.

\section{NOTES}

1. Ilse Aichinger, Brüder Grimm, Der Wolf und die sieben jungen Geißlein, Wien, Edition Korrespondenzen, 2004. Abréviation: W7G.

2. Ilse Aichinger, «Der Wolf und die sieben jungen Geißlein », in: Jochen Jung (Hrsg.): Märchen, Sagen und Abenteuergeschichten auf alten Bilderbogen neu erzählt von Autoren unserer Zeit, München, Heinz Moos Verlag, 1974, p. 9. Abréviation: JJ.

3. Il est à noter que ce texte est repris par Helmut Brackert pour figurer dans l'anthologie du conte publiée en 1994: Helmut Brackert (Hrsg.), Das große deutsche Märchenbuch, München, Zürich, 
Artemis und Winkler, 1994.

Le texte d'Ilse Aichinger présenté p. 861 est inclus dans la section « Kunstmärchen ».

4. Ilse Aichinger, Die größere Hoffnung, Frankfurt am Main, Fischer Taschenbuch Verlag, ${ }^{6} 1999$. Abréviation: GH.

5. Max Lüthi, Märchen, zweite Auflage, Stuttgart, J.B. Metzlersche Verlagsbuchhandlung, MCMLXIV, p. 12.

6. Sur cette page figurent sept gravures monochromes d'A. Pock et un texte abrégé du conte.

7. Richard Reichensperger (1961-2004) a été responsable de l'édition en 8 volumes des œuvres complètes d'Ilse Aichinger chez Fischer Taschenbuch Verlag en 1991. Il est à noter que son décès survient six ans exactement après la disparition de Clemens, le fils d'Ilse Aichinger mort dans des circonstances analogues.

8. Ilse Aichinger, Unglaubwürdige Reisen, Frankfurt am Main, S. Fischer Verlag, 2005, p. 153. Abréviation: UR.

9. E. M. Cioran, De l'inconvénient d'être né, Paris, Gallimard, coll. « Folio essais », 2008, p. 66.

10. Marthe Robert, Roman des origines et origines du roman, Paris, Gallimard, coll. «Tel », 1990, p. 197. Abréviation: RO.

11. Michel Pastoureau, Dictionnaire des couleurs de notre temps. Symbolique et société, Paris, Éditions Bonneton, 1992, pp. 199-203.

I. Aichinger mentionne d'ailleurs cette ambivalence dans une note de son Journal, datée de 1950, in: Kleist, Moos, Fasane, Frankfurt am Main, Fischer Taschenbuch Verlag, 1991, p. 44. Abréviation: KMF.

12. Georges Jean, Le pouvoir des contes, Paris, Castermann, coll. «E3 », 1981, p. 187.

13. Bruno Bettelheim, Psychanalyse des contes de fées, Paris, Éditions Robert Laffont, 1976, p. 197. Abréviation : PCF.

14. Die Märchen der Brüder Grimm. Kinder -und Hausmärchen, München, Wilhelm Goldmann Verlag, 1964, pp. 33-35. Abréviation: MG.

15. «Polster » est substitué à « Kissen » et « Nachtmahl » à " Abendbrot ».

16. Vladimir Propp, Morphologie du conte, Paris, Ed. du Seuil, ${ }^{3} 1973$, p. 123. Abréviation: VP.

17. Il est à noter que la ligne de séparation entre les paragraphes n'existe pas dans le livre de Jochen Jung.

18. Traduction de Jean-François Boutout, in: Ilse Aichinger, Eliza Eliza, Lagrasse, Verdier, coll. « Der Doppelgänger », 2007, pp. 308-309. Abréviation: E.E.

«Das Erzählen in dieser Zeit», in: Ilse Aichinger, Der Gefesselte, Frankfurt am Main, Fischer Taschenbuch Verlag, 1991, p. 10. Abréviation: G.

Voir à ce sujet Sylvaine Faure-Godbert, «Vom Ende her auf das Ende hin erzählen. Die Poetik des Endes im Erzählband Der Gefesselte von Ilse Aichinger», in: I. Rabenstein-Michel/ F. Rétif/ E. Tunner (Hrsg.), Ilse Aichinger. Misstrauen als Engagement?, Würzburg, Königshausen \& Neumann, 2009, pp. 99-108.

19. Ilse Aichinger, Subtexte, Wien, Edition Korrespondenzen, ${ }^{2} 2006$, p. 13 . Abréviation: $S$.

20. Cette notice est rédigée dans l'année qui suit la mort de Günter Eich.

21. Manuel Esser, «Die Vögel beginnen zu singen, wenn es noch finster ist », in: Samuel Moser (Hrsg.), Ilse Aichinger. Leben und Werk, Frankfurt am Main, Fischer Taschenbuch Verlag, 1995, p. 50 .

22. Traduction de Marthe Robert, in: Grimm: Märchen. Contes, Choix de contes traduits de l'allemand, préfacés et annotés par Marthe Robert, Paris, Gallimard, 1990, p. 17. Abréviation : MC. 23. Le regard de l'enfance est célébré dans l'essai consacré à Ernst Schnabel en 1952 et à la perspective de l'étrangeté qu'il développe dans ses écrits, "Die Sicht der Entfremdung ", in: Ilse Aichinger, Kurzschlüsse. Wien (Courts-circuits. Vienne), Wien, Edition Korrespondenzen, 2001, pp. 51-62. Abréviation: KW. 
24. Ces conflits irrésolus trouvent par ailleurs un écho discret dans les poèmes, récits et pièces radiophoniques. Voir à ce sujet I. Rabenstein-Michel, « Vater/Figuren bei Ilse Aichinger », in: Ilse Aichinger. Misstrauen als Engagement?, op. cit., pp. 130-145.

25. Ilse Aichinger, «Ich halte meine Existenz für völlig unnötig», un entretien avec Julia Kospach, in: Profil, Nr. 45/2003 (UR, 181-187). Le leitmotiv du «premier cri » (de désespoir) du nouveau-né qui plonge la mère dans le ravissement sillonne de nombreux textes tardifs.

26. Les questions posées par le docteur Mengele lors de sa visite dans l'appartement familial à Vienne en représentent la forme extrême. In: Film und Verhängnis. Blitzlichter auf ein Leben (Cinéma et destin tragique. Flashes d'une vie), Frankfurt am Main, Fischer Verlag, 2001, p. 56. Abréviation: FV.

27. Elle part avec l'un des derniers transports d'enfants organisés par les Quakers.

28. Le sujet est traité de façon métaphorique dans le récit de 1952 "Wo ich wohne » ("Là où j'habite », G, 93-98), voir Brigitte Desbrière-Nicolas, « Die versunkene Arche », in: Ilse Aichinger. Misstrauen als Engagement?, op. cit., pp. 53-62.

29. Helga Michie, Concord, Wien, Edition Korrespondenzen, 2006.

30. Traduction: J.F. Boutout, op. cit., E.E., p. 309.

31. Brigitta Oesch, «Schiffbruch des Erzählens: Eine intertextuelle Lektüre der RotkäppchenVersion in Ilse Aichingers Roman Die größere Hoffnung» in: Zeitschrift für Semiotik, 2002, Bd. 24, p. 277-293.

32. B. Desbrière-Nicolas, «Engel in der Nacht. L'ange dans la prose de jeunesse d'Ilse Aichinger», in: Germanica, 1999, n²4, pp. 117-135. B. Desbrière-Nicolas, «Le déluge dans Die größere Hoffnung d'Ilse Aichinger », in : Femmes et tradition du Livre, Lille, Université Charles-de-Gaulle, coll. « UL3 », 2006, pp. 39-47.

33. Cette idée est défendue par E. Fried. Erich Fried, «Die größere Hoffnung », in: S. Moser, op. cit., pp. 155-156.

34. Voir à ce propos Irene Heidelberger-Leonard, «Klärung oder Verklärung? Überlegungen zu Ilse Aichingers Roman Die größere Hoffnung», in: Heidy Margrit Müller (Hrsg.), Verschwiegenes Wortspiel. Kommentare zu den Werken Ilse Aichingers, Bielefeld, Aisthesis Verlag, 1999, pp. 157-168.

35. La jeune I. Aichinger l'a bien compris: l'appartenance à une communauté qui persécute impitoyablement les faibles, libère de la peur de la solitude et de la mort et étouffe les interrogations désespérées relatives à la perte du sens de l'existence. Voir B. Desbrière-Nicolas, «Die versunkene Arche », op. cit., p. 56.

36. Albert Camus, Lettres à un ami allemand, Paris, Gallimard, Folio, 2006.

37. B. Oesch, op. cit., p. 291.

38. Extrait d'un entretien publié dans le Berner Zeitung (22.12.1984). Brita Steinwendtner, «Ein Paar Fragen in Briefen - Gespräch mit Ilse Aichinger», in : Kurt Bartsch, Gerhard Melzer (Hrsg.), Ilse Aichinger, Graz, Literaturverlag Droschl, 1993,p. 12.

\section{RÉSUMÉS}

En 2004, Ilse Aichinger publie à Vienne un opuscule au titre peut-être trop familier qui rencontre peu d'écho dans la critique : Le loup et les sept chevreaux. L'oeuvre qui ne compte qu'une vingtaine de pages n'a pourtant rien d'anodin. Déjà le propos introductif où s'enchevêtrent le souvenir de la petite enfance passée à Linz et l'affirmation de l'attitude négative cultivée par l'écrivain 
vieillissant face à l'existence, révèle en filigrane l'intention qui sous-tend la mise en œuvre du conte relevée dès le premier roman de 1948, Le plus grand espoir. L'article montre qu'Ilse Aichinger attribue subrepticement à chacun des différents textes constituant l'opuscule de 2004 (propos introductif relatif au premier livre de contes, texte des frères Grimm, puis sa réécriture singulière) une fonction déterminante. L'œuvre tardive ouvre la voie au déchiffrement de la transcription littéraire énigmatique d'une histoire personnelle douloureuse, manifestée dès le roman des débuts consacré au cataclysme qui a ébranlé le siècle. Le plus grand espoir fait l'objet d'une analyse qui s'appuie sur la réflexion de Marthe Robert relative à l'origine du genre romanesque et à ses affinités avec le conte. Cette deuxième partie de l'étude met en évidence l'art de l'utilisation du conte et sa dimension subversive, destinée à mettre au jour les désordres d'un monde absurde.

2004 erschien in Wien ein Buch von I. Aichinger, das trotz... oder wegen seines allzu vertraut klingenden Titels wenig beachtet wurde: Der Wolf und die sieben jungen Geißlein. Die kaum fünfundzwanzig Seiten umfassende Publikation ist kein harmloses Spiel mit einer tradierten Erzählform. Schon der einleitende Text, der auf dem Zusammenspiel von Erinnerung an die frühe Kindheit in Linz und Spiegelung der für die alternde Schriftstellerin charakteristischen düsteren Grundeinstellung zur Existenz beruht, lässt die Absicht erkennen, die seit dem Erstlingsroman Die größere Hoffnung (1948) beim Einsatz von Märchen verfolgt wird. Im vorliegenden Aufsatz wird gezeigt, wie jedem der einzelnen Texte im Buch vom Jahre 2004 (einleitende Betrachtungen zum „Grüne[n] Märchenbuch aus Linz“, Grimm-Text und neue eigenartige Version) unauffällig eine Schlüsselrolle zugewiesen wird. So gibt das Spätwerk Anlass zu einer Dechiffrierung der rätselhaften literarischen Verarbeitung einer lebenslangen Leidensgeschichte, wie sie sich schon im Frühroman manifestiert, der die Katastrophe des Jahrhunderts zum Gegenstand hat. Die größere Hoffnung wird anhand Marthe Roberts Überlegungen zum Ursprung der Romangattung und ihrer Beziehung zum Märchen untersucht. Dadurch wird unter anderem der kunstvolle Einsatz des Märchens anschaulich gemacht sowie seine subversive Dimension, die zur Aufdeckung der Mißstände in einer als sinnlos empfundenen Welt beitragen soll.

In 2004 Ilse Aichinger published an opuscule in Vienna entitled: The Wolf and the Seven Little Kids. This title was perhaps a little too familiar to attract much critical attention. Nevertheless, this work of some twenty pages is far from insignificant. The introductory remarks intertwine the memory of an early childhood spent in Linz with the assertion of the negative attitude to existence developed by the ageing author, immediately hinting at the intention which underlies the implementation of this fairy tale, and which could be noted in her first novel published in 1948, Herod's Children. The article shows how Ilse Aichinger surreptitiously attributes a determining function to each of the different texts which constitute the 2004 opuscule (introductory remarks relating to her first book of tales, the Grimm brothers' text, and then her singular rewriting). This work, written late in life, opens the way to the deciphering of the enigmatic literary transcription of a painful personal history, a history which was already present in the early novel devoted to the cataclysm that shook the century. Herod's Children is subjected to an analysis based on Marthe Robert's reflection relating to the origin of the novelistic genre and its affinities with the fairy tale. The second part of this study reveals the art of the use of the fairy tale and its subversive dimension, destined to bring to light the disorders of an absurd world. 
AUTEUR

BRIGITTE DESBRIÈRE-NICOLAS

Université Charles-de-Gaulle Lille 3 